\title{
Adaptation of Hybrid FSO/RF Communication System Using Puncturing Technique
}

\author{
Muhammad Nasir KHAN ${ }^{1}$, Mohsin JAMIL ${ }^{2}$, Mazhar HUSSAIN ${ }^{3}$ \\ ${ }^{1}$ Department of Electrical Engineering, The University of Lahore, Pakistan \\ 2 Department of Electrical Engineering, The Islamic University in Madinah, Saudi Arabia \\ ${ }^{3}$ Department of Computer Science, Institute of Southern Punjab, Pakistan \\ muhammad.nasir@ee.uol.edu.pk,mohsin@iu.edu.sa,mazhar.hussain@isp.edu.pk
}

Manuscript received June 04, 2016

\begin{abstract}
Spectrum of radio frequency $(R F)$ communications is limited and expensive to install new applications. Free space optical (FSO) communication is a viable technology which offers enormous bandwidth, license free installation, inexpensive deployment and error prone links. The FSO links degrade significantly due to the varying atmospheric and weather conditions (fog, cloud, snow, haze and combination of these). We propose a hybrid FSO/RF communication system which adapts the varying nature of atmosphere and weather. For the adaption of varying atmosphere and weather scenarios, we develop a novel optimization algorithm. The proposed algorithm is based on the well-known puncturing technique. We provide an extrinsic information transfer (EXIT) chart for the binary and quaternary mapping scheme for the proposed communication system. We simulate the proposed algorithm for the hybrid communication system and analyze the system performance. The proposed algorithm is computationally less expensive and provide better performance gains over varying atmosphere and weather conditions. The algorithm is suitable for fast speed applications.
\end{abstract}

\section{Keywords}

Free Space Optical (FSO) communication, Low Density Parity Check (LDPC) code, Extrinsic Information Transfer (EXIT), Puncturing Optimization Algorithm (POA), Minimum Positive Difference (MPD)

\section{Introduction}

Communicating over FSO, with its nearly boundless bandwidth, has been proposed as viable candidate for "last mile connectivity" [1]. But the meteorological conditions and scintillation severely affect the FSO channel. FSO communication undergoes significant deterioration whenever the visibility of the laser beam is affected. It is mentioned in [2] that fog is the dominant parameter which significantly affects the visibility of the FSO laser beam. The atmospheric effects on the FSO beam propagation can be divided into power attenuation and laser power fluctuation usually called scintillation [2]. Atmosphere effects on the FSO link have been investigated in [2-4]. These channel variations can be improved by introducing the rate adapting technique. We have investigated in the previous chapters that we can achieve certain gains by implementing the coding scheme over the signal independent Gaussian noise model. Previously, we specifically concentrated on the improved performance of the system by implementing LDPC codes. But we did not investigate the rate adaptability of the LDPC codes. Therefore, it is a good idea to investigate the implication of rate adaptability of LDPC codes by implementing the puncturing techniques.

Previous study on punctured LDPC codes can be found in [5], [6], considering the binary erasure (BEC) channels. Low density parity check (LDPC) code was first introduced by Gallager [7] in 1962 and its performance is nearly close to the Shannon's limits. The LDPC codes of any rate and block length can also be created easily by just changing the shape of the parity check matrix. It means that the rate adaptability in the LDPC codes can be obtained easily compared to other codes. LDPC codes have the feature of parallelism for supporting different speeds, performances and memory consumption. It is therefore better to design LDPC codes because of the capacity approaching performance and comparable easy implementation [8]. Extrinsic information transfer (EXIT) chart [9] is a good approach to measure the code convergence performance. It consists of two components decoder curves, variable and check node curves. We will utilize this approach as a performance measure in optimization problem.

Recent research [2], [6] shows that the demerits of one communication links (FSO) can be overcome by introducing a parallel channel of other communication link (RF) named as hybrid FSO/RF communications system. But a true hybridization can be made possible only when both channels collaboratively compensate the disadvantages of each other 
and providing good performance improvements of the communication system. To cope with the channel variations, we propose puncturing LDPC codes which provides better performance improvement by making the system adaptive over the non-adaptive (fixed) system [6]. The proposed algorithm shows a better flexibility over a slowly fading channels.

These codes have the flexibility of operating over a wide range of code rate having a same encoder and decoder pair. Having a single pair of encoder and decoder is the main aim of the hybrid code design which can adapts the weather variations by introducing the puncturing algorithm. We propose a novel optimization algorithm which implements rate adaptability of LDPC code over a wide range of rates. It optimizes the puncturing fractions for different weather conditions (clear sky, rain and fog). We show that transmission reliability over a range of rate is possible using the proposed algorithm as compared to a fixed system in [6]. We are providing investigations on how the puncturing algorithm adapts different weather conditions in a flexible fashion using quadrature modulation scheme. Our objective is to provide transmission reliability over a wide range of rate for the hybrid communication system by adapting the optimized puncturing patterns for varying meteorological conditions. Our puncturing scheme is different from the existing puncturing scheme [10] that we are proposing the scheme which can be applicable for a generalized channel model (single, hybrid and block fading). We investigate over different weather scenarios in hybrid channel model using the binary and quaternary modulation. The proposed algorithm provides a better adaptation over a wide range of rate for different channels.

\section{Irregular LDPC Codes Preleminary}

Low density parity check (LDPC) codes have received attention by the research community because of their capacity approaching performance over a large range of data transmission and storage channels. The original Gallager codes are known to be regular LDPC codes [7]. For another class of codes known as irregular LDPC codes, Luby et al. [11] demonstrated that the properly constructed codes can achieve better performance comparing to the regular ones.

An LDPC code can be well represented by a bipartite graph known as Tanner graph [11]. The Tanner graph consists of two sets of component nodes decoders known as variable nodes decoder (VND) and check nodes decoder (CND). The number of edges which join the VND and CND are equal to the number of $1 \mathrm{~s}$ in the sparse parity check matrix $\mathcal{H}$. The iterative decoding of the LDPC code is performed by passing messages between the neighboring VND and CND. The flow of information between the variable and check nodes is shown in Fig. 1.

Irregular LDPC structures [12] are those for which the degrees of each set of nodes are chosen according to some distribution. For a right regular also known as check-regular LDPC structure, its all check nodes have the same degree. For

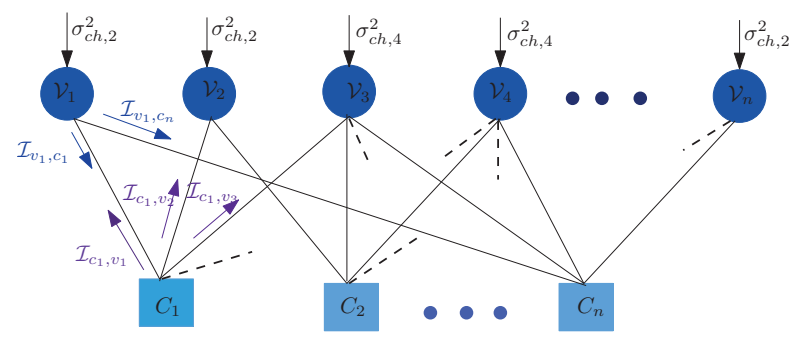

Fig. 1. Tanner graph representation of irregular LDPC codes and exchange of information flow between the CND and VND.

irregular LDPC code structure, we can define a degree distribution ensemble $(\lambda, \rho)$ from edge perspective way as [8]

$$
\begin{aligned}
& \lambda(x)=\sum_{i=d_{v}^{\min }}^{d_{v}^{\max }} \lambda_{i} x^{i-1}, \\
& \rho(x)=\sum_{i=d_{c}^{\min }}^{d_{c}^{\max }} \rho_{i} x^{i-1}
\end{aligned}
$$

where $d_{v}^{\min }$ and $d_{v}^{\max }$ are the minimum and maximum variable node degrees respectively, $d_{c}^{\min }$ and $d_{c}^{\max }$ are the minimum and maximum check node degrees respectively and the fraction of edges which are connected to degree $i$ variable nodes is denoted by $\lambda_{i}$ and the fraction of edges which are connected to degree $i$ check nodes, is denoted by $\rho_{i}$. The set of variable (check) nodes of degree $i$ (equivalently columns(rows) of weight $i$ ) shall be designated by $\mathcal{V}_{i}\left(C_{i}\right)$ and translating from edge perspective to node perspective as

$$
\begin{gathered}
\mathcal{V}_{i}=\frac{\frac{\lambda_{i}}{i}}{\sum_{j=d_{v}^{\min }}^{d_{\max }^{\max }} \frac{\lambda_{j}}{j}}, \\
C_{i}=\frac{\frac{\rho_{i}}{i}}{\sum_{j=d_{v}^{\min }}^{d_{\max }^{\max }} \frac{\rho_{j}}{j}} .
\end{gathered}
$$

According to [13], for a fixed check node degree (rightregular), if $\lambda_{i}$ be the edge fraction incident to variable node of degree $d_{v_{i}}$, then we can find the variable node fraction $v_{i}$ of degree $i$ using the following expression as well,

$$
v_{i}=\frac{\left(1-R_{m}\right) d_{c}}{d_{v_{i}}} \times \lambda_{i}
$$

where $R_{m}$ denotes the mother code rate. For the rest of the chapter we denote the block length by $n$, the number of check nodes by $m$, the number of edges by $E$.

\section{Puncturing Prelaminaries}

An error correcting code can be considered to be the rate-adaptive codes when the information rate of the code is dynamically adapted to the communication channel requirement. We investigate the feature of rate-adaptability of the irregular LDPC codes by introducing the puncturing technique. Puncturing increases the rate of originally constructed code, (i.e., $C(n, k)$ ), by deleting a set of symbols from the codeword, where $p<n$. It then converts the code ensemble 
(i.e., $C(n, k))$ into a new code ensemble $C(n-p, k)$. The punctured coding rate $R_{p}$ is then increased to

$$
R_{p}=\frac{k}{n-p}
$$

where (6) can be written in the context of puncturing fraction as

$$
R_{p}=\frac{R_{m}}{1-p}
$$

where $p$ represent the puncturing fraction and can be calculated for a given code rate using (7). We can further defined it with new name "overall puncturing" because we are dividing the puncturing distribution into the different population type, i.e., population type means different variable node degree. Then the overall puncturing fraction $\mathcal{P}$ can be defined as

$$
\mathcal{P}=\frac{1}{\varrho} \sum_{i} p_{i} v_{i}
$$

where $\varrho$ is variable node population types in the irregular LDPC code structure. In order to find the optimum puncturing fractions, we propose an efficient puncturing algorithm and verify the results using the Monte Carlo simulations.

\subsection{VND and CND EXIT Functions}

EXIT chart was first introduced in [14] for measuring convergence behavior of iteratively decoded parallel concatenated codes and later on generalized for LDPC codes analysis in [13], [15], [16]. The main components of the EXIT chart are the EXIT functions of the component decoders which relates the a-priori mutual information (MI) available to the component decoder which then generates the extrinsic MI after iterative decoding.

Considering the Gaussian assumption of the incoming messages to a variable node $v$ of degree $i$, the EXIT function for the code component involving the variable nodes for irregular structure is given by [13]

$$
\mathcal{I}_{E V}\left(\mathcal{I}_{A V}, \sigma_{\mathrm{ch}}^{2}\right)=\sum_{i} \lambda_{i} \mathcal{J}\left(\sqrt{(i-1)\left[\mathcal{J}^{-1}\left(\mathcal{I}_{A V}\right)\right]^{2}+\sigma_{\mathrm{ch}, v}^{2}}\right)
$$

where $\mathcal{J}$ has been defined in [9], [14], $\sigma_{\text {ch, } v}^{2}=\frac{4}{\sigma_{n}^{2}}$ for the unpunctured variable node $v\left(\sigma_{n}^{2}\right.$ denotes the channel noise variance), $\sigma_{\mathrm{ch}, v}^{2}=0$ for the punctured variable nodes $v$ and the Fig. 1 is redrawn as Fig. 2. Similarly the EXIT function for the check node decoder involving all the check nodes for irregular structure can be written as

$$
\mathcal{I}_{E C}\left(\mathcal{I}_{A C}\right)=1-\sum_{i} \rho_{i} \mathcal{J}\left(\sqrt{i-1} \times \mathcal{J}^{-1}\left(1-\mathcal{I}_{A C}\right)\right)
$$

\subsection{Puncturing Optimization (PO) Algorithm}

Our objective is to provide transmission reliability over a wide range of rate by adapting the optimized puncturing patterns for varying weather conditions. The criteria is to

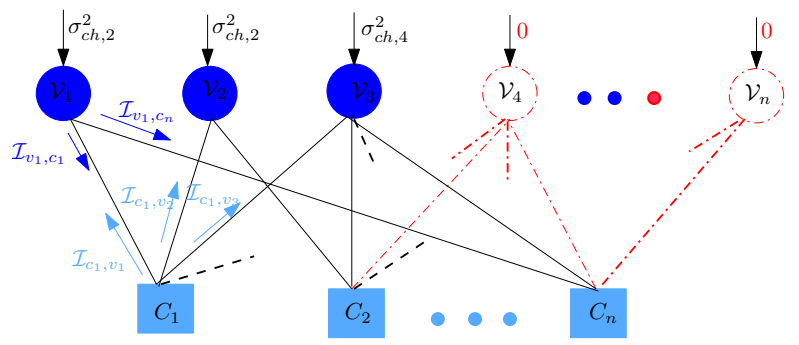

Fig. 2. Tanner graph representation of irregular LDPC codes with punctured and unpunctured variable nodes.

find the best threshold by optimizing the puncturing patterns for varying weather conditions over a wide range of rates. In the proposed algorithm, Puncturing Optimization (PO), we define the Minimum Positive Difference (MPD) rule which calculates the minimum positive value when the two EXIT curves obtained using (9) and (10) just touches.

The PO algorithm can be summarized in the following algorithmic steps as

1. Define $\gamma$ and $\widehat{\gamma}$ (i.e., varying weather condition in terms of $\kappa)$ and puncturing code rate.

2. Initialize the fine/course grid search method and calculate $p$ using (7), calculate different puncturing patterns using (8) for each desired rate and ensure the puncturing fractions for variable nodes should be between 0 and 1. Algorithm is flexible in terms of speed and efficiency which is dependent on the grid search method (fine/coarse).

3. Initialize average signal to noise ratio (SNR) $(\bar{\gamma})$, which gives the average MI. Then transmit the average MI through the communication channel (single/hybrid/block fading) depending on respective weather conditions.

4. Perform numerical calculation for EXIT curves using (9) and (10) for each of the puncturing combinations and given SNR.

5. Calculate the MPD between the two curves, if we got the positive value MPD where the tunnel between the EXIT curves opens, we accept those values (puncturing patterns and corresponding thresholds) otherwise again go to Step 3 to increase the average SNR. This step should run for each defined puncturing pair in Step 2 . The MPD can be expressed mathematically as,

$$
\begin{cases}\operatorname{MPD} & : \min _{\mathcal{I}_{A V}}\left(\mathcal{I}_{E V}\left(\mathcal{I}_{A V}\right)-\mathcal{I}_{E C}^{-1}\left(\mathcal{I}_{A V}\right)\right) \\ \text { s.t } & : \operatorname{MPD}>\epsilon_{\mathrm{thr}}\end{cases}
$$

where we select the threshold $\epsilon_{\mathrm{thr}}=2 \times 10^{-3}$ which is very small value to give accurate enough results.

6. Select the best optimum puncturing pairs $\mathcal{P}_{i}$ and the corresponding thresholds $\{(\gamma, \widehat{\gamma})\}$ over a particular weather scenarios. 
7. For each defined rate, pickup the best minimum threshold and the corresponding puncturing patterns obtained from Step 6 for varying weather conditions.

8. Repeat all above steps for varying weather conditions of $\delta$.

We extend (9) in the context of puncturing fraction and the new extrinsic MI can be expressed as,

$$
\mathcal{I}_{E V}\left(\mathcal{I}_{A V}, \sigma_{\mathrm{ch}}^{2}\right)=\sum_{i} \lambda_{i}\left[p_{i} \mathcal{J}(\sqrt{\psi})+\left(1-p_{i}\right) \xi\right]
$$

where $\psi=(i-1)\left[\mathcal{J}^{-1}\left(\mathcal{I}_{A V}\right)\right]^{2}$ and $\xi=\mathcal{J}\left(\sqrt{\psi+\sigma_{\mathrm{ch}, i}^{2}}\right)$.

Since we are puncturing the variable nodes so that (10) will remain same. In order to find the optimum puncturing fraction and the corresponding threshold, we run the proposed algorithm defined in Sec. 3. Although we did simulation for a wide range of rate $R_{p}=0.3 \rightarrow 0.8$ but we are showing one simulation results in Fig. 3 for a given rate of $R_{p}=0.6$. The pink arrow over the variable node curve shows that the distance between the two EXIT curves decreases and we end-up with an optimized results. We are presenting few puncturing patterns and the corresponding threshold obtained using the proposed algorithm in Tab. 1 for three cases.

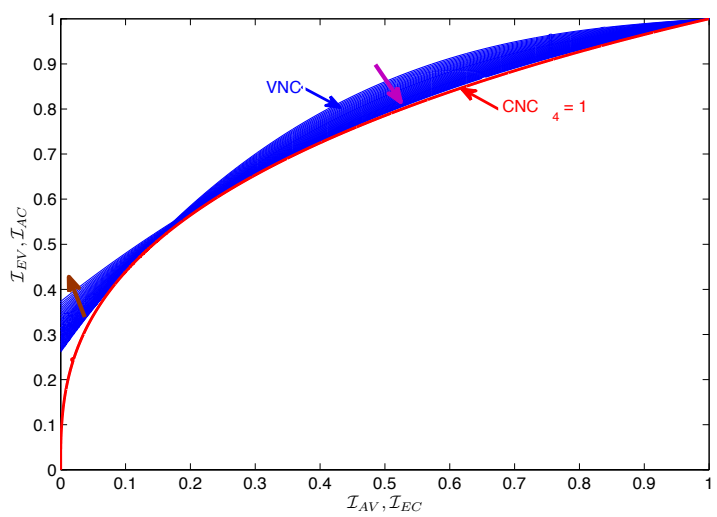

Fig. 3. Code optimization using EXIT functions of VND and CND using the proposed algorithm.

\begin{tabular}{|l|l|l|}
\hline \multicolumn{3}{|c|}{ Puncturing Fractions $p_{2}=0.5 p_{4}, p_{2}=p_{4}, p_{4}=0.5 p_{2}$} \\
\hline \multirow{2}{*}{$R_{p}=0.3, p=0.17, \mathrm{C}=-0.62 \mathrm{~dB}$} & $p_{2}$ & {$[0.11,0.17,0.23]$} \\
& $p_{4}$ & {$[0.23,0.17,0.11]$} \\
& $\gamma[\mathrm{dB}]$ & {$[0.80,0.75,0.65]$} \\
\hline \multirow{2}{*}{$R_{p}=0.4, p=0.38, \mathrm{C}=-0.24 \mathrm{~dB}$} & $p_{2}$ & {$[0.25,0.38,0.51]$} \\
& $p_{4}$ & {$[0.51,0.38,0.25]$} \\
& $\gamma[\mathrm{dB}]$ & {$[1.50,1.30,1.10]$} \\
\hline \multirow{3}{*}{$R_{p}=0.5, p=0.5, \mathrm{C}=0.19 \mathrm{~dB}$} & $p_{2}$ & {$[0.34,0.50,0.66]$} \\
& $p_{4}$ & {$[0.66,0.50,0.34]$} \\
\hline \multirow{3}{*}{$R_{p}=0.6, p=0.58, \mathrm{C}=0.68 \mathrm{~dB}$} & $p_{4}[\mathrm{~dB}]$ & {$[2.20,1.80,1.45]$} \\
\hline \multirow{2}{*}{$R_{p}=0.7, p=0.64, \mathrm{C}=1.23 \mathrm{~dB}$} & $p_{2}$ & {$[0.39,0.58,0.77]$} \\
& $p_{4}[\mathrm{~dB}]$ & {$[3.77,0.58,0.39]$} \\
& $\gamma[\mathrm{dB}]$ & {$[0.43,0.64,0.85]$} \\
\hline \multirow{2}{*}{$R_{p}=0.8, p=0.69, \mathrm{C}=2.04 \mathrm{~dB}$} & $p_{2}$ & {$[0.65,0.64,0.43]$} \\
& $\gamma[\mathrm{dB}]$ & {$[0.72,0.69,2.20]$} \\
\hline
\end{tabular}

Tab. 1. Optimum puncturing fraction.

\section{Hybrid FSO/RF Channel}

We are implementing the proposed algorithm over a hybrid channel (FSO/RF) and verify that the proposed algorithm achieves transmission reliability by adapting the rate in each channel depending the respective channel weather scenarios. We did not see such analysis before and by using the proposed algorithm, we can increase system effectiveness using a single pair of encoder and decoder. Our objective is to decrease the computational complexity and cost of the communication system using single pair of encoder and decoder considering a slow varying channel. We are considering the additive white Gaussian noise (AWGN) channel and varying the channel conditions by $\delta$, where the value of $\delta$ denotes the channel quality i.e., either clear sky condition or bad channel condition. The detailed block diagram of the hybrid FSO/RF coding is shown in Fig. 4. At the transmitter side, the system inputs $k$-length information bits, which are encoded by rate $R_{p}=\frac{R_{m}}{1-\mathcal{P}}$.

\subsection{FSO Channel Model}

The FSO link employs intensity modulation with direct detection using two level-pulse amplitude modulation (2-PAM) transmission scheme. The received signal $(y)$ after the optical to electrical conversion is given by

$$
y=\eta P x+z
$$

where $\eta$ is the photodetector efficiency which is assumed to be unity for simplicity, $x \in\{0,1\}$ is the transmitted optical symbols after puncturing, $P^{2}$ is the optical received signal to noise ratio $(\gamma)$. Various noise models are possible including Poisson, signal dependent Gaussian and signal independent Gaussian noise [1]. In this paper, we use the signal independent Gaussian noise model, where $z$ is the Gaussian distributed random variable with zero mean and unit variance.

\subsection{RF Channel Model}

We consider a line of sight (LOS) RF channel which is modelled as a fading free AWGN channel. Let $\widehat{P}$ be the transmit RF power on the RF link, then the received RF noisy signal $\widehat{y}$

$$
\widehat{y}=\sqrt{\widehat{P}} \widehat{x}+\widehat{z}
$$

where $\widehat{x} \in\{+1,-1\}$ is the transmitted RF symbols after puncturing using binary phase shift keying (BPSK), $\widehat{z}$ is the AWGN with zero mean and unit variance. Quaternary mapping scheme is implemented in Sec. 5. The received RF SNR is defined as $\widehat{\gamma}=\sqrt{\widehat{P}}$.

\subsection{Puncturing Code Design}

In a hybrid FSO/RF communication system, our aim is to transmit data bits over two independent parallel channels 


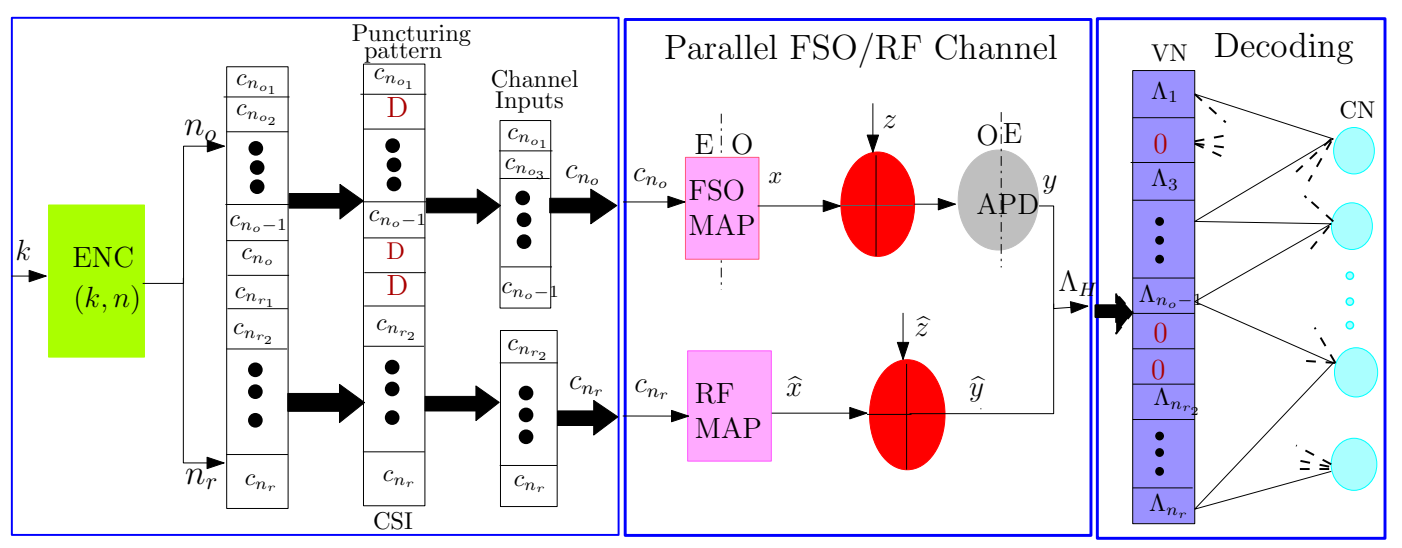

Fig. 4. Detailed Block diagram of hybrid FSO/RF coding system.

(hybrid FSO/RF). Previously researchers [17] have suggested a separate error-correcting code for each channel. Here, however, our main goal is to design only one LDPC code as shown in Fig. 4. It is noted that the channel state information (CSI) is available at the transmitter so that the appropriate puncturing ratio can be selected. We have shown an example of puncturing pattern in which $D$ denotes the position of punctured (deleted) bits. The ratio of the puncturing will be determined by the CSI. Each $n$-bit codeword after puncturing is split into 2 streams of lengths $n_{o}$ and $n_{r}$ to be transmitted on FSO and RF links respectively. The resulting blocks are sent through the respective channel, i.e., $c_{n_{o}}$ coded bits through the FSO link and $c_{n_{r}}$ bits through the RF links respectively. Puncturing will be suitable in order to incorporate different weather conditions. It will puncture the bits depending on weather condition and adapts the rate of transmission in a flexible fashion.

In hybrid channel case, we use the Tanner graph representation to define the ensemble $C(\Psi, \rho)$ of bipartite graphs. We represent $E$ be the set of edges in the graph and let $E_{o}$ and $E_{r}$ be the set of edges that are incident with variable nodes corresponding to the optical and RF channels, respectively. We can extend (1) in terms of hybrid channel representation as, $\lambda_{o}(x)=\sum_{i} \lambda_{i}^{o} x^{i-1}$ and $\lambda_{r}(x)=\sum_{i} \lambda_{i}^{r} x^{i-1}$, where $\lambda_{i}^{o}\left(\lambda_{i}^{r}\right)$ is the fraction of edges connected the optical (RF) variable node of degree $i$. We are considering the right regular LDPC code structure, so (2) will remain same. Then the $\Psi$ in the new code ensemble for the hybrid code design can be designated as $\Psi=\left\{\lambda^{o}(x), \lambda^{r}(x)\right\}$. In the hybrid code design, we assume that the number of RF punctured nodes is given by $n \hat{p}$, where $\widehat{p}$ is the fraction of RF nodes that are punctured. In the same way, we denote the FSO punctured nodes by $n p$ and $p$ is the fraction of punctured FSO variable nodes.

\section{Implementing POA Using Quater- nary Modulation}

We begin by discussing how the EXIT chart based techniques of [9], [13] can be used to optimize the LDPC code using the binary modulated AWGN channels. Our ob- jective here is to implement the proposed algorithm using quaternary modulations (QPSK/4-PAM). The block diagram shown in Fig. 4 of the conventional system can be extended to Fig. 5 to incorporate the mapper/de-mapper construction. The coded bits from the transmitter are then permuted using interleaver to generate the interleaved codeword. The codeword vector is then fed to the mapper that converts the bits into symbols. Each symbol is either complex or real depending on the type of mapper (QPSK/4-PAM). The symbols are normalized to have average energy $\widehat{\mathcal{E}}_{s}=\frac{1}{M} \sum_{i=1}^{M}\left|\widehat{x}_{i}\right|^{2}$ for QPSK and $\mathcal{E}_{s}=\frac{1}{M} \sum_{i=1}^{M} x_{i}$ for 4-PAM, where $M=2^{m}$ and $m$ is the number of bits in each symbol.

We then extend the algorithm for the quaternary modulations scheme for the hybrid FSO/RF channel. For this we analyze the demapper EXIT curves for different mapping schemes (Gray and anti-Gray). We provide investigations for 4-PAM and QPSK EXIT over varying channel conditions. Then we simulate the overall EXIT chart curve for the hybrid FSO/RF channel. Since we consider the parallel channel, we also measure the best possible fraction of bits to be transmitted to the respective channel.

The main components of the receiver are the demapper and the LDPC decoder, each of which is implemented using the log likelihood ratio (LLR) values. Conventionally they are connected with interleavers and deinterleavers [18] but in our simulation, we did not implement the interleaver and deinterleavers because of the randomness created by the

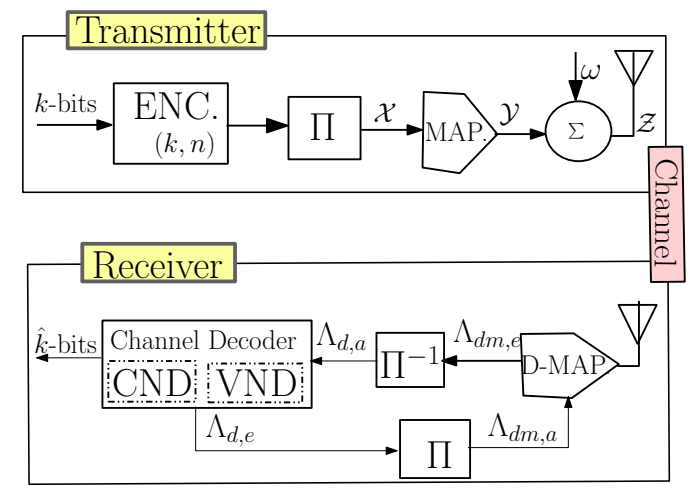

Fig. 5. Implementation of quaternary modulation for the hybrid FSO/RF communication system. 
variable node decoder itself. The received symbols are passed into the demapper, which demaps the symbols and produce a vector $\Lambda_{d m, e}$ known as the extrinsic LLR of the demapper. The vector $\Lambda_{d m, e}$ is then deinterleaved by $\Pi^{-1}$ and the resulting vector $\Lambda_{d, a}$ is fed in to the LDPC decoder known as LDPC a priori LLR which works on standard sum product algorithm. The output of the LDPC decoder $\Lambda_{d, e}$ is then fed back to the interleaver and the resulting vector $\Lambda_{d m, a}$ is introduced as the input to the demapper known as demapper a priori LLR. This is known as the iterative process between the demapper and the LDPC decoder and shown in Fig. 6.

\subsection{Demapper Soft Value}

The demapper is based on soft value operation and it extracts the soft value of each coded bit. In the softdemapper, the channel symbols $\mathcal{Z} \in\left\{y_{1}, y_{0}\right\}$ for the FSO channel are demapped in the form of LLR $\Lambda_{d m, e}$. Here in our analysis, we are considering both the QPSK(Gray/antiGray) and 4-PAM(Gray/anti-Gray), each symbol represented by two coded bits (i.e., for FSO channel $x_{1}, x_{0}$ and for RF channel $\widehat{x}_{1}, \widehat{x}_{0}$ ), the demapper needs to calculate the LLR of the coded bits for each incoming channel symbol. For the sake of simplicity and to save space, we provide formulation only for the FSO coded bits $\left(x_{1}, x_{0}\right)$. The LLR value of $x_{0}$ conditioned on the matched filter output $\mathcal{Z}$ can be calculated using the Bayes's rule as

$$
\begin{aligned}
& \Lambda\left(x_{0} \mid \mathcal{Z}\right)=\Lambda_{a}\left(x_{0}\right)+ \\
& \ln \frac{p\left(\mathcal{Z} \mid x_{0}=1, x_{1}=0\right)+p\left(\mathcal{Z} \mid x_{0}=1, x_{1}=1\right) \cdot \mathrm{e}^{\left(\Lambda_{a}\left(x_{1}\right)\right)}}{p\left(\mathcal{Z} \mid x_{0}=0, x_{1}=0\right)+p\left(\mathcal{Z} \mid x_{0}=0, x_{1}=1\right) \cdot \mathrm{e}^{\left(\Lambda_{a}\left(x_{1}\right)\right)}} .
\end{aligned}
$$

For an arbitrary number of $M$ coded bits $x_{x_{0}, \cdots, M-1}$ per symbol $\mathcal{Z}$, we can write the LLR values of $k$-bits as [19]

$$
\begin{aligned}
\Lambda\left(x_{k} \mid \mathcal{Z}\right) & =\underbrace{\Lambda_{a}\left(x_{k}\right)}_{\text {a priori }} \\
+ & \underbrace{\sum_{i=0}^{\sum^{M-1}-1} p\left(\mathcal{Z} \mid x_{k}=1, x_{j, j=0, \cdots, M-1, j \neq k}\right) \cdot \mathrm{e}^{\sum_{j=0, j \neq k}^{M-1} \Lambda_{a}\left(x_{j}\right)}}_{\text {extrinsic }}
\end{aligned}
$$

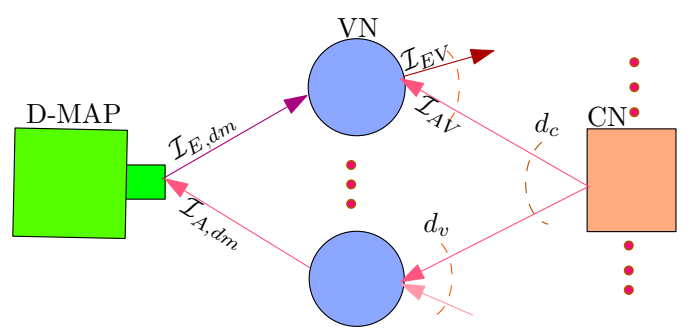

Fig. 6. Iterative graph of demapper and LDPC decoder.

\subsection{Transfer Characteristics of Demapper}

The shape of the transfer characteristics of the demappers QPSK/4-PAM depends on the channel SNR and bit labeling pattern pattern (Gray/anti-Gray) [20]. We then proceed by showing the performance comparisons of the demappers depending on the bit mapping by calculating the EXIT curves. We measured the demappers's EXIT curve by running a number of Monte Carlo simulation [18]. The demappers's EXIT function can be represented as $\mathcal{I}_{E, d m}\left(\mathcal{I}_{A, d m}, \gamma\right)$. The results of the Monte Carlo simulations for the demapper transfer curves are shown in Fig. 7.

From the simulation results in Fig. 7, it is noticed that Gray mapping doesn't show any improvement while the antiGray mapping is showing improvement after a number of iteration. The information transfer curve for Gray mapping is not going to change for increasing a priori knowledge and it stays fairly constant. It shows the strong impact of selecting the mapping scheme iterative process. However the anti-Gray mapping scheme is showing some slope with increasing the a priori knowledge.

\subsection{Overall EXIT Chart Using POA}

In our work we proposed the adaptation of POA over the joint channel conditions (e.g., we consider two situations, clear sky condition for delta $(\delta$ stands for the channel quality) equals to zero and bad weather condition for delta equal to 0.25 ) and try to minimize the positive difference between the two EXIT chart curves. The POA elaborate for the hybrid FSO/RF communication system. We evaluate the POA over a wide range of rate and it shows from the results that the algorithm is adaptive over all the rates. Furthermore the algorithm is adaptive over varying channel conditions. We propose POA and measure the EXIT chart for the given code rate. The EXIT chart measures the mutual MI jointly and gives an estimate of code convergence. While measuring the MI, we introduce a new parameter named as delta and say that the quality of both channels will be controlled by delta. In this way, we use two values of delta in simulations which incorporate different weather conditions.

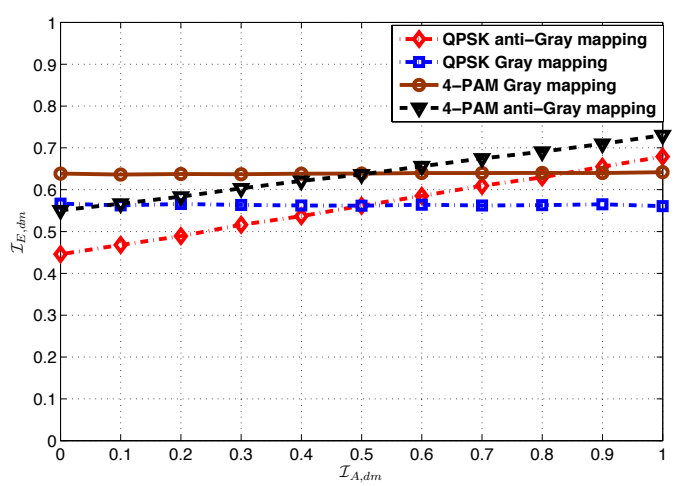

Fig. 7. EXIT chart curves for QPSK/4-PAM demappers for $\mathrm{SNR}=1.0 \mathrm{~dB}$ using the AWGN a-priori channel approximation. 


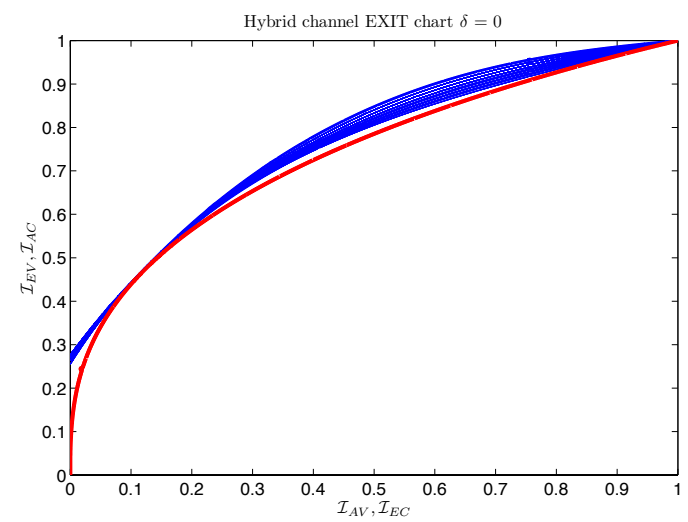

(a) EXIT Chart at $\delta=0$ and $\left(R_{p}=0.7\right)$.

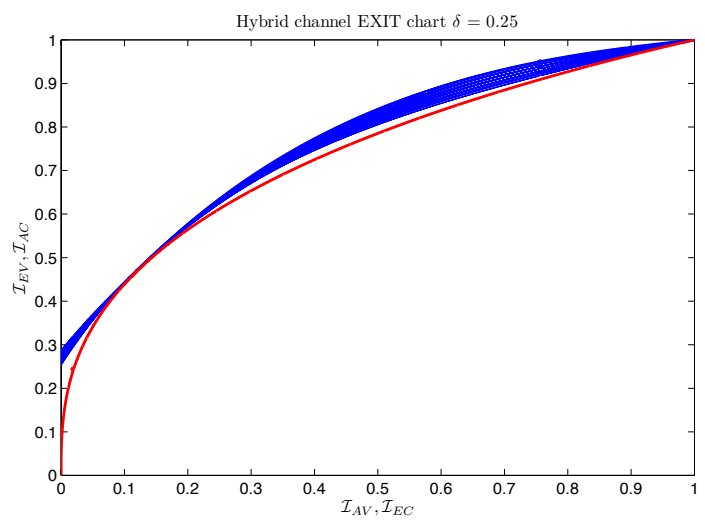

(b) EXIT Chart at $\delta=0.25$ and $\left(R_{p}=0.7\right)$.

Fig. 8. Best Threshold (i.e., optimized puncturing pair for the best value of SNR).

Our objective is to optimize the code using the puncturing technique and we provide example for the binary mapping. We know that the optimization criteria in our proposed algorithm requires two EXIT curves to be generated, one that corresponds to the VND, we call $\mathcal{I}_{E V}$ and another that corresponds to the CND, we call $\mathcal{I}_{E C}$. The goal is then fit the two curves together by picking the appropriate puncturing ratios (patterns). It is seen from Fig. 5, that the VND curve characterizes not only the variable nodes of the LDPC code, but also the characteristics curve of the mapping. The overall VND EXIT curve is created by first generating the transfer characteristics curve for the mapper at the given SNR which is known as the demapper EXIT curve. The demapper curve can be generated using Monte Carlo simulation [14], [18] under the assumption that the demapper's a priori input is conditionally Gaussian [9]. We can extend (9) in the form of overall quaternary modulation VND EXIT function as

$$
\begin{aligned}
\mathcal{I}_{E V} & \left(\mathcal{I}_{A V}, \gamma, d_{v}\right)=p \mathcal{J}(\sqrt{\kappa}) \\
& +(1-p) \mathcal{J}\left(\sqrt{\kappa+\left[\mathcal{J}^{-1}\left(\mathcal{I}_{E, d m}\left(\mathcal{I}_{A, d m}, \gamma\right)\right)\right]^{2}}\right)
\end{aligned}
$$

where $\kappa=\left(d_{v}-1\right)\left[\mathcal{J}^{-1}\left(\mathcal{I}_{A V}\right)\right]^{2}, \mathcal{I}_{E, d m}\left(\mathcal{I}_{A, d m}, \gamma\right)=$ $\mathcal{J}\left(\sqrt{\left.d v \cdot \mathcal{J}^{-1}\left[\left(\mathcal{I}_{A, d m}\right)\right]^{2}+\sigma_{c h}^{2}(\gamma)\right)}\right)$ and we can model the a-priori LLR, i.e., $\mathcal{I}_{A, d m}\left(\mathcal{I}_{A V}, d_{v}\right)=\mathcal{J}\left(\sqrt{d v} \cdot \mathcal{J}^{-1}\left(\mathcal{I}_{A V}\right)\right)$, for the demapper which is the output of the VND and is assumed as Gaussian random variable [13]. It is noted that (17) represents the regular LDPC code VND curve. In case of an irregular curve, we can extend (17) as

$$
\begin{aligned}
\mathcal{I}_{E V}\left(\mathcal{I}_{A V}, \gamma\right)= & \sum_{i=1}^{d v_{\max }} v_{i}\left\{p_{i} \mathcal{I}_{E V}\left(\mathcal{I}_{A V}, \gamma, d_{v_{i}}\right)\right. \\
& \left.+\left(1-p_{i}\right) \mathcal{I}_{E V}\left(\mathcal{I}_{A V}, 0, d_{v_{i}}\right)\right\} .
\end{aligned}
$$

It is noticed from Fig. 7, that QPSK with Gray mapping, we cannot have any mapping gain and therefore, we can utilized (18) for analytical simulations. The simulation results

\begin{tabular}{|c|c|c|c|c|c|c|c|}
\hline \multicolumn{10}{|c|}{ Puncturing Optimization Algorithm Results (QPSK) } \\
\hline$\delta$ & $R_{p}$ & $p_{2}$ & $p_{4}$ & $\widehat{p}_{2}$ & $\widehat{p}_{4}$ & $\gamma[\mathrm{dB}]$ & $\widehat{\gamma}[\mathrm{dB}]$ \\
\hline \hline 0 & 0.7 & 0.96 & 0.32 & 0.96 & 0.32 & -0.675 & -0.675 \\
\hline \hline 0.25 & 0.7 & 0.96 & 0 & 0.96 & 0.64 & 0.175 & -3.16 \\
\hline
\end{tabular}

Tab. 2. Puncturing fraction for each rate in hybrid FSO/RF channel (QPSK).

showing the behavior of EXIT chart variations (optimization) for variable puncturing ratios. The results obtained for the hybrid channel are shown in Fig. 8 and presented in Tab. 2 .

If $\delta=0$ as given in Tab. 2, weather is considered to be clear and it will not affect the MI of the system. On the other hand, if the value of $\delta \neq 0$ (delta $=0.25$ as given in Tab. 2), it means that the weather is not clear and the MI is affected by a given amount of delta. So this is the way, we can incorporate different weather condition in simulation. It is also technically very clear that if the weather is clear, the system performs well with maximum MI and on the other way around for bad weather, the performance of the system is also worse with low MI.

\section{Conclusion}

We propose a novel puncturing optimization algorithm for rate adaptation and analyse its performance for the hybrid FSO/RF channel case. We see that the propose algorithm well adapted the different weather scenarios by providing the best threshold and the corresponding puncturing patterns. It distribute the amount of channel bits in an efficient way under equal and unequal channel conditions in a flexible fashion. We see that our proposed algorithm performs well under all channel conditions and provide better performance. New results for the hybrid EXIT chart have been derived for the binary and quaternary modulation schemes as well which present the behavior of EXIT chart in the hybrid channel. We also develop the analysis for the quaternary modulation and provide the simulation results of the corresponding mappers. The new research results will help in implementing the proposed algorithm for high speed communication applications. The algorithm is computationally less complex and efficient. 


\section{Acknowledgments}

The author would like to thank Prof. Bill Cowley and Dr. Khoa D. Nguyen from the Institute for Telecommunication Research, South Australia for providing helpful support and useful suggestions during the course of investigation.

\section{References}

[1] GAGLIARDI, R. M., KARP, S., Optical Communications. 2nd ed. New York (USA): John Wiley \& Sons, Inc., 1995. ISBN: 978-0-471-54287-2

[2] KHAN, M. N., COWLEY, W., NGUYEN, K. Link adaptation of FAHOR communication system. In Proceedings of the Australian Communications Theory Workshop (AusCTW). Wellington (New Zealand), 2012, p. 120-125. DOI: 10.1109/AusCTW.2012.6164917

[3] MAKKI, B., SVENSSON, B., ERIKSSON, T., et al. On the performance of RF-FSO links with and without Hybrid ARQ. IEEE Transactions on Wireless Communications, 2016, vol. 15, no. 7, p. 49284943. DOI: $10.1109 /$ TWC.2016.2549537

[4] KHAN, M. N. Importance of noise models in FSO communications. EURASIP Journal of Wireless Communication and Networking, Feb. 2014, vol. 2014, no. 102, p. 1-10. DOI: 10.1186/1687-14992014-102

[5] PISHRO-NIK, H., FEKRI, F. Results on punctured low density paritycheck codes and improved iterative decoding techniques. IEEE Transactions on Information Theory, 2007, vol. 53, no. 2, p. 599-614. DOI: 10.1109/TIT.2006.889701

[6] KHAN, M. N., JAMIL, M. Maximizing throughput of free space communications system using puncturing technique. Arabian Journal for Science and Engineering, Nov. 2014, vol. 39, no. 12, p. 8925-8933. DOI: $10.1007 / s 13369-014-1451-6$

[7] GALLAGER, R. Low-density parity-check codes. IRE Transactions on Information Theory, 1962, vol. 8, no. 1, p. 21-28. DOI: 10.1109/TIT.1962.1057683

[8] JOHNSON, S. Iterative Error Correction: Turbo, Low-Density Parity-Check and Repeat-Accumulate Codes. New York: Cambridge University Press, 2010. ISBN: 9780521871488

[9] HAGENAUER, J. The EXIT chart-introduction to extrinsic information transfer in iterative processing. In Proceeding of the 12th European Signal Processing Conference (EUSIPCO). Vienna (Austria), Sep. 2004, p. 1541-1548. ISBN: 978-320-0001-65-7

[10] ESLAMI, A., VANGALA, S., PISHRO-NIK, H. Hybrid channel codes for efficient FSO/RF communication systems. IEEE Transaction on Communication, Oct. 2010, vol. 58, no. 10, p. 2926-2938. DOI: 10.1109/TCOMM.2010.082710.090195

[11] LUBY, M., MITZENMACHER, M., SHOKROLLAHI, M., et al. Improved low-density parity-check codes using irregular graphs. IEEE Transactions on Information Theory, Feb. 2001, vol. 47, no. 2, p. 585598. DOI: $10.1109 / 18.910576$
[12] RICHARDSON, T., URBANKE, R. The capacity of low density parity-check codes under message-passing decoding. IEEE Transactions on Information Theory, 2001, vol. 47, no. 2, p. 599-618. DOI: $10.1109 / 18.910577$

[13] TEN BRINK, S., KRAMER, G., ASHIKHMIN, A. Design of lowdensity parity-check codes for modulation and detection. IEEE Transactions on Communications, Apr. 2004, vol. 52, no. 4, p. 670-678. DOI: 10.1109/TCOMM.2004.826370

[14] TEN BRINK, S. Convergence behavior of iteratively decoded parallel concatenated codes. IEEE Transactions on Communications, Oct. 2001, vol. 49, no. 10, p. 1727-1737. DOI: 10.1109/26.957394

[15] SHARON, E., ASHIKHMIN, A., LITSYN E. Analysis of lowdensity parity-check codes based on EXIT functions. IEEE Transactions on Communications, Jul. 2006, vol. 54, no. 7, p. 1407-1414. DOI: 10.1109/TCOMM.2006.877935

[16] ASHIKHMIN, A., KRAMER, G., TEN BRINK, S. Extrinsic information transfer functions: model and erasure channel properties. IEEE Transactions on Information Theory, Nov. 2004, vol. 50, no. 11, p. 2657-2673. DOI: 10.1109/TIT.2004.836693

[17] TAPSE, H., BORAH, D. Hybrid optical/RF channels: characterization and performance study using low density parity check codes. IEEE Transactions on Communications, 2009, vol. 57, no. 11, p. 3288-3297. DOI: 10.1109/TCOMM.2009.11.080170

[18] RAKIA, T., YANG, H.-C., ALOUINI, M.-S., et al. Outage analysis of practical FSO/RF hybrid system with adaptive combining. IEEE Communications Letters, Aug. 2015, vol. 19, no. 8, p. 1366-1369. DOI: 10.1109/LCOMM.2015.2443771

[19] TEN BRINK, S., SPEIDEL, J., YAN, R.-H. Iterative demapping and decoding for multilevel modulation. In Proceedings of the Global Telecommunications Conference. 1998, vol. 1, p. 579-584. DOI: 10.1109/GLOCOM.1998.775793

[20] TEN BRINK, S. Designing iterative decoding schemes with the extrinsic information transfer chart. AEU International Journal of Electronics and Communications, 2000, vol. 54, no. 6, p. 389-398.

\section{About the Authors ...}

Muhammad Nasir KHAN was born in Sialkot, Pakistan. He received his M.Sc. from TU Delft in 2009 and Ph.D. from the Institute for Telecommunication Research, South Australia. His research interests include signal processing for communication, channel coding and detection.

Mohsin JAMIL was born in Gujranwala, Pakistan. He received his M.Sc. from NUS, Singapore and Ph.D. from University of Southampton, UK. His research interests include control system and robotics.

Mazhar HUSSAIN was born in Multan, Pakistan. He received his Ph.D. from France. His research interests include image and signal processing. 